\title{
Efeito do transtorno depressivo sobre os rendimentos do trabalho por gênero e setor produtivo brasileiro
}

\author{
Alan Marques Miranda Leal ${ }^{1}$ \\ Evandro Camargos Teixeira ${ }^{2}$
}

\begin{abstract}
RESUMO
O objetivo desse estudo é verificar o efeito do transtorno depressivo sobre os rendimentos do trabalho por sexo e setor de atividade no Brasil. Para tal, utilizou-se o modelo de Mínimos quadrados em dois estágios (MQ2E) com dados da Pesquisa Nacional por Amostra de Domicílios (PNAD) para o ano de 2008. Como principais resultados, verifica-se que os rendimentos variam de negativo (esperado) a positivo em relação a incidência do transtorno depressivo sobre os indivíduos. Cabe destacar, por exemplo, que os rendimentos de homens acometidos por essa enfermidade no setor de serviços domésticos são 176,22\% menores em relação aos trabalhadores desse mesmo setor, mas sem a doença. No caso das mulheres, verificou-se que ser acometida pelo transtorno depressivo e trabalhar no setor industrial implica em diminuição da ordem de $91,02 \%$ nos rendimentos em relação às mulheres que trabalham no mesmo setor, contudo não foram diagnosticadas com o referido transtorno.
\end{abstract}

Palavras-chave: transtorno depressivo, rendimentos do trabalho, mínimos quadrados em dois estágios.

\section{Effect of depressive disorder on work income by gender and Brazilian productive sector}

\begin{abstract}
The objective of this study is to verify the effect of depressive disorder on income from work by sex and sector of activity in Brazil. For this, the Least Squares model in two stages (MQ2E) was used with data from the National Household Sample Survey (PNAD) for 2008. As main results, it appears that the yields vary from negative (expected) to positive regarding the incidence of depressive disorder on individuals. It is worth noting, for example, that the earnings of men affected by this disease in the domestic services sector are $176.22 \%$ lower in relation to workers in the same sector, but without the disease. In the case of women, it was found that being affected by depressive disorder and work in the industrial sector implies a decrease of $91.02 \%$ in income in relation to women who work in the same sector, however they were not diagnosed with the referred disorder.
\end{abstract}

Keywords: depressive disorder, work income, two-stage least squares.

\section{INTRODUÇÃO}

Segundo a Organização Mundial da Saúde (WHO, 2017), o transtorno depressivo é uma doença caracterizada pela tristeza, perda de interesse ou prazer, cansaço, sentimento de culpa e pouca concentração, atingindo mais de 320 milhões de pessoas em todo o mundo.

Entre as causas do transtorno depressivo, Lederbogen et al. (2011) consideram que a intensa urbanização das sociedades modernas, com pouco tempo de descanso e muita pressão em relação ao trabalho, contribui para o desenvolvimento e aumento do número de casos dessa enfermidade. Além disso, há características hereditárias que tornam algumas pessoas mais suscetíveis ao desenvolvimento da referida doença (SOBOCKI et al., 2006).

Diante das causas elencadas do transtorno depressivo, percebe-se seu caráter multidisciplinar. Enquanto, inicialmente, a Psicologia e a Medicina se interessaram por esse transtorno e seu tratamento, há muitas outras Ciências que posteriormente passaram também a analisar causas e consequências da doença, tais como a Sociologia, a Economia, dentre outras.

Para a Economia, o interesse advém das suas consequências sobre a capacidade produtiva do indivíduo, sua qualidade de vida e a de seu próprio país. Nesse sentido, a teoria

\footnotetext{
${ }^{1}$ Mestre em Economia/UFMG. Email: alanmarquesleal@ hotmail.com

${ }^{2}$ Professor Associado I. Departamento de Economia/UFV. Email: evandro.teixeira@ufv.br
} 
econômica postula que o transtorno depressivo pode impactar tanto o nível de salários dos indivíduos quanto o crescimento econômico do país. A primeira conexão diz respeito ao fato de que indivíduos ao sofrerem um episódio depressivo são mais suscetíveis ao presenteísmo e absenteísmo. Presenteísmo diz respeito à perda de produtividade do indivíduo em seu trabalho devido à doença. Já o absenteísmo corresponde a infrequência do indivíduo em seu ofício, dada a enfermidade. Como ambos os fenômenos diminuem sua produtividade, eles impactam, por conseguinte, em seus rendimentos.

A segunda conexão se refere ao fato de que o indivíduo acometido pelo transtorno depressivo tem menores incentivos para se qualificar, quando comparado ao indivíduo saudável. Isso advém do fato de que a pessoa com o transtorno depressivo não pode ofertar mão de obra no mercado de trabalho com a mesma frequência e quantidade que uma pessoa sem esse transtorno. Isso prejudica no longo prazo a capacidade de crescimento do país, dado que o acúmulo do capital humano atua tanto como facilitador de inovações quanto do aprendizado, aumentando a produtividade dos trabalhadores na economia e, portanto, seu crescimento (MANKIW et al., 1992, LUCAS,1988, e ROMER, 1990).

Além dos efeitos supracitados, é importante salientar que as parcelas da população acometidas pelo transtorno depressivo são diferenciadas em termos de gênero e setores produtivos. Com relação ao primeiro aspecto, a literatura aponta que existe cerca de duas mulheres com a doença para cada homem acometido (BAPTISTA et al., 1999; AUBE et al., 2000; JUSTO e CALIL, 2006; CORREIA e BORLOTI, 2011).

No que tange o segundo aspecto, a partir da PNAD de 2008 é possível afirmar que cerca de $6,45 \%$ dos trabalhadores domésticos possuem o diagnóstico ${ }^{3}$ da doença, enquanto no caso do setor da construção tal percentual cai para apenas $2,1 \%$. Nesse sentido, cerca de $3,7 \%$ dos trabalhadores agrícolas e da indústria de transformação foram diagnosticados com transtorno depressivo, enquanto esse percentual aumenta para 4,3\% no caso dos funcionários da administração pública.

Essas discrepâncias implicam não apenas no diferencial de perdas em termos de rendimentos, mas também prejudicam a própria disponibilidade de mão de obra na economia. Assim, o mercado de trabalho de setores que demandam maior qualificação, tal como indústria de transformação, estão especialmente suscetíveis a variações no acometimento pela doença sobre os ofertantes da mão de obra. Dado um cenário de pleno emprego, a referida doença pode prejudicar o crescimento de um ou mais setores da economia.

Diante dos impactos mencionados, o presente trabalho tem como objetivo analisar o efeito do transtorno depressivo sobre os rendimentos do trabalho, considerando possíveis diferenciais existentes entre os gêneros e diferentes setores da economia. A abordagem é incipiente na literatura internacional e nacional, não sendo encontrado nenhum trabalho que tivesse objetivo similar ao do presente estudo. Além desta seção, este trabalho se divide em mais quatro seções, a seguir: Aspectos Teóricos e Empíricos, Procedimentos metodológicos, Resultados e Discussões, e Considerações Finais.

\section{ASPECTOS TEÓRICOS E EMPÍRICOS}

Não existe um modelo teórico específico para o problema de pesquisa específico tratado aqui. O modelo microeconômico de saúde e rendimentos usado no presente estudo é uma adaptação do modelo desenvolvido originalmente por Grossman (1972). Esse modelo considera

\footnotetext{
${ }^{3}$ A PNAD 2008 considera apenas o diagnóstico de transtorno depressivo, ou seja, é necessário que o indivíduo tenha o parecer de um psiquiatra. Observa-se que tal metodologia desconsidera os sintomas da doença ou mesmo os casos não-diagnosticados, o que pode contribuir para a subestimação de sua ocorrência no país.
} 
que um indivíduo qualquer maximiza sua utilidade ao longo da vida. Tal utilidade depende dos bens que ele consome, sua saúde e também seu tempo de lazer. Em termos matemáticos:

$$
\mathrm{U}=\mathrm{U}(\mathrm{C}, \mathrm{H}, \Phi)
$$

Em que $\mathrm{C}$ são os bens consumidos pelo indivíduo, $\mathrm{H}$ é seu estoque (estado) de saúde e $\Phi$ é seu lazer. O estado de saúde do indivíduo é função dos bens que ele consome ao longo de sua vida $(\mathrm{C})$, exclusive os bens relacionados ao cuidado da saúde, representados separadamente pela variável Y; de um conjunto de outras variáveis exógenas $(Z)$ e também de U, que representa outras variáveis não-captadas diretamente pelas explicitadas na equação. Assim, segundo Grossman (1972), a função de produção saúde é dada por:

$$
\mathrm{H}=\mathrm{H}(\mathrm{C}, \mathrm{Y}, \Phi, \mathrm{Z}, \mathrm{U})
$$

Um indivíduo ao longo de sua vida, contudo, não maximiza sua função de utilidade sem nenhuma restrição. Seus gastos em saúde e bens diversos devem igualar seu estoque inicial de riqueza e seu fluxo de pagamentos em um mesmo período. Em termos práticos, isso é dado pela equação abaixo:

$$
\sum \frac{P_{i} M_{i}+V_{i} X_{i}}{(1+r)^{i}}=\sum \frac{W_{i} T W_{i}}{(1+r)^{i}}+A_{0}
$$

Em que $\mathrm{r}$ é taxa de juros de desconto dos investimentos do indivíduo, $\mathrm{M}$ e $\mathrm{X}$ são, respectivamente, serviços médicos e os demais bens consumidos, com $\mathrm{P}$ e $\mathrm{V}$ sendo seus preços, $\mathrm{W}$, seu salário-hora e TW, o tempo de trabalho.

Observa-se que o indivíduo também se submete às restrições relacionadas ao seu tempo. Ele pode tanto dispender tempo em atividades relacionadas ao mercado (TW) quanto a atividades relacionadas ao não-mercado $(\mathrm{TH})$, aproveitar o tempo com lazer (T) ou mesmo não realizar nenhuma outra atividade devido a alguma doença (TL) - nesse caso, o transtorno depressivo.

$\mathrm{TW}+\mathrm{TL}+\mathrm{TH}+\mathrm{T}=\Omega$

Em que TH é tempo saudável dispendido em atividades não-relacionadas ao mercado nem ao lazer e $\Omega$ é o tempo total do indivíduo.

Por fim, o tempo disponível do indivíduo é todo aquele no qual ele não é acometido pelo transtorno depressivo, ou seja:

$\mathrm{TL}=\Omega-\mathrm{h}_{\mathrm{i}}$

É importante ressaltar que devido a expressão (5), o indivíduo enfrenta um trade-off entre trabalho e lazer. Em termos práticos, isso indica que o transtorno depressivo atua como redutor de tempo de dispêndio também em relação as atividades relacionadas ao trabalho. Esse trade-off advém do fato de que o indivíduo troca seu tempo dispendido em qualquer atividade, exclusive o trabalho, por tempo nessa atividade.

Assim, quanto mais o indivíduo trabalha, mais recursos ele tem disponível para despender em bens de produção externa, ou seja, efetivamente adquirir bens no mercado. $\mathrm{O}$ transtorno depressivo afeta essa relação na medida em que diminui o tempo disponível para a realização de qualquer outra atividade, fazendo que sua produtividade do trabalho caia, assim como, por consequência, seus rendimentos. Logo, a teoria apresentada viabiliza a relação esperada pelo presente trabalho, qual seja: a doença diminui o tempo que o indivíduo aloca para o trabalho, assim como sua produtividade.

No que tange a literatura, como supracitado, são raros os trabalhos realizados. Um desses estudos foi o de Bós e Bós (2005), que avaliaram o efeito do transtorno depressivo na qualidade de vida dos idosos, verificando que existe relação inversamente proporcional.

Cai et al. (2006) analisaram o impacto da doença sobre a empregabilidade de trabalhadores australianos. Utilizando-se da Household, Income and Labor Dynamics in Australia Survey (HILDA), os autores concluíram que o declínio na qualidade da saúde (autodeclarada) de um indivíduo australiano diminui a probabilidade de este estar empregado, independente do sexo e nível de qualidade da saúde. Como exemplo, os autores verificaram que 
para homens com idade superior a 50 anos, uma redução na qualidade de saúde de boa (good) para razoável (fair) reduz a probabilidade de estes estarem no mercado de trabalho em quase $7 \%$.

O único trabalho relacionado à temática realizado no Brasil foi o de Benedicto (2017). A autora utilizou a PNAD de 2008 a fim de verificar o impacto do transtorno depressivo sobre as remunerações de homens e mulheres, concluindo que a relação causal é endógena, isto é, a referida doença tanto diminui as remunerações das mulheres, quanto o baixo rendimento as torna mais suscetíveis a esse transtorno. A conclusão do estudo é que o transtorno depressivo de fato reduz os rendimentos dos indivíduos, sendo essa redução da ordem de $13,11 \%$ para homens e $78,1 \%$ para mulheres.

\section{PROCEDIMENTOS METODOLÓGICOS}

O presente estudo estimará equações de rendimentos, adaptando-as de forma a permitir a inclusão da variável de interesse do presente trabalho, qual seja a existência do transtorno depressivo e a interação desta com os setores da economia. As equações mincerianas serão estimadas através do Método dos Mínimos Quadrados Ordinários (MQO) e Mínimo Quadrados em dois Estágios (MQ2E), mediante a existência de endogeneidade.

\subsection{Equação de Mincer e MQO}

A equação de rendimentos é uma formulação de Mincer (1974), ancorada na teoria do Capital Humano. Tal formulação considera que os rendimentos dos indivíduos dependem da educação formal destes e de suas experiências (proxy de treinamento em serviço). Espera-se que os rendimentos aumentem em função da elevação dessas duas variáveis, sendo que a elevação com a experiência ocorre a taxas decrescentes. Tendo em vista tais relações e a literatura empírica das equações de rendimentos, a equação que viabiliza empiricamente o presente trabalho é, conforme Wolff (2009), a seguinte:

$$
\ln R_{E, X}=\ln R_{0}+r E+r^{\prime} k_{0} X-\left(\frac{r^{\prime k_{0}}}{2 T}\right) * X^{2}
$$

Em que $\mathrm{R}$ é o salário do indivíduo, com $R_{0}$ sendo seu rendimento inicial, E é o número de anos de escolaridade, $\mathrm{X}$ é sua experiência e $\mathrm{r}$ é o retorno à escolaridade.

As estimações são realizadas geralmente via Mínimos Quadrados Ordinários (MQO). Tal método de estimação é largamente utilizado em estimações de diversas amostras. O MQO consiste na minimização da soma dos quadrados dos resíduos. Assim, busca-se minimizar a seguinte equação:

$$
\sum u_{i}^{2}=\Sigma\left(Y_{i}-\beta_{0}-\beta_{1} X_{1}\right)^{2}
$$

Ao se encontrar os coeficientes que minimizem tal equação, tem-se, conforme, Wooldridge (2002), que eles são $B L U E^{4}$. É importante mencionar algumas suposições importantes referentes ao MQO, tais como ausência de multicolinearidade e autocorrelação, homocedasticidade, exogeneidade, dentre outras. A hipótese de exogeneidade é de especial interesse para o presente trabalho e consiste basicamente no fato de a variável explicativa $X$ independer da variável dependente Y. Essa suposição é pouco robusta para o presente trabalho, visto que o nível de rendimentos do indivíduo pode impactar em suas chances de desenvolver

\footnotetext{
${ }^{4}$ Better Linear Unbiased Estimator, ou seja, Melhor Estimador Linear Não-Tendecioso, caracterizado tanto pelo não-viés quanto pela eficiência.
} 
ou não o transtorno depressivo. Matematicamente, a quebra da hipótese de exogeneidade é dada por:

$$
\operatorname{cov}\left(X_{i} \mid u_{i}\right) \neq 0
$$

$\mathrm{O}$ que indica basicamente que a variável explicativa $\mathrm{X}$ e os resíduos se comportam semelhantemente, ou seja, de modo não-aleatório. Os coeficientes encontrados via MQO estão, dessa forma, contaminados, isto é, os supostos efeitos parciais não são bem captados.

Exibe-se adiante a equação a ser estimada via MQO (equação 9), sustentadas as hipóteses anteriores, inclusive a de exogeneidade. Cabe salientar que as variáveis explicativas selecionadas tiveram como critério a literatura concernente ao tema.

$$
\begin{gathered}
\ln \left(\text { salarioh }_{i}\right)=\beta_{0}+\beta_{1} \text { educ }_{i}+\beta_{2} \text { exper }_{i}+\beta_{3} \text { exper }_{i}{ }_{i}+\beta_{4} \text { branco }_{i}+ \\
\beta_{5} \text { formal }_{i}+\beta_{6} N_{i}+\beta_{7} \text { Ne }_{i}+\beta_{8} \text { Co }_{i}+\beta_{9} \text { Sul }_{i}+\beta_{10} \text { urban }_{i}+\beta_{11} \text { chefe }_{i}+
\end{gathered}
$$

$\sum_{i=12}^{19} \quad \beta_{i}$ atividade $_{i}+\sum_{i=19}^{26} \quad \beta_{i}$ atividade $_{i} *$ depr $+\mu_{i}$

Em que $\ln ($ salarioh) é a variável dependente correspondente ao logaritmo da renda do trabalho principal; educ são os anos de estudo; exper é a experiência, calculada através da seguinte fórmula: idade - anos de estudo - 6; exper $^{2}$ é a experiência elevada ao quadrado; branco é uma dummy que recebe valor 1 se o indivíduo é branco e 0 caso contrário; formal também é uma dummy para auferir se os trabalhadores possuem carteira assinada, recebendo valor 1 se o trabalhador é formal e 0 caso contrário; N, Ne, Co, Sul e Se (categoria de referência) são dummies regionais, que representam as regiões norte, nordeste, centro-oeste, sul e sudeste, respectivamente; urban se refere a uma dummy que recebe valor 1 se o indivíduo reside na zona urbana e 0 se reside na zona rural; chefe denota a posição do indivíduo dentro do domicílio, assumindo valor igual a 1, caso ele seja a pessoa de referência, e 0 , caso contrário; atividade representa os oito setores produtivos considerados no presente estudo: agricultura, indústria, comércio, serviços coletivos, serviços domésticos, transportes, administração pública e outras atividades (categoria de referência); e atividade*depr é uma dummy de interação entre as dummies que denotam os oito setores produtivos e aquela que aponta se o indivíduo foi diagnosticado com depressão.

Cabe ainda salientar que Santos e Kassouf (2007) denotam que as mulheres são mais suscetíveis a depressão em relação aos homens. Diante disso, os resultados serão apresentados por sexo, de modo a captar os efeitos específicos segundo o gênero, objetivo específico do estudo.

Por fim, a suposição de exogeneidade será testada tanto para homens quanto para mulheres. Caso ela seja corroborada, pode-se proceder o uso do modelo MQO, porém se essa suposição for violada, deve-se implementar alguma solução para tal problema. A correção mais utilizada é o método das variáveis instrumentais (VI) ou também conhecido como Mínimos Quadrados em Dois Estágios (MQ2E).

\subsection{Mínimos Quadrados em Dois Estágios (MQ2E)}

O MQ2E consiste na utilização de um instrumento ou mais instrumentos como preditor(es) da(s) variável(is) explicativa(s) endógena(s) ao sistema. Um instrumento é uma variável exógena ao sistema, que explica com certa precisão (a ser detalhada mais adiante) a variável independente endógena ao sistema. Segundo Wooldridge (2002), cria-se uma nova variável (no presente estudo, Depr, por exemplo), a partir de outras variáveis que expliquem relativamente bem a variabilidade desta. Caso os instrumentos sejam satisfatórios $\left(\mathrm{R}^{2}\right.$ relativamente elevado e teste $\mathrm{F}$ significativo), então substitui-se a variável transtorno depressivo original do banco de dados pela predita pelos instrumentos. 
Para verificar a possível endogeneidade entre uma variável explicativa e a dependente, é necessária a aplicação de um teste de endogeneidade, sendo mais utilizado o teste de Hausman (1978). Nesse sentido, utilizando-se o referido teste e logo em seguida o MQ2E (se necessário), a seguinte equação será estimada:

Depr $_{i}=\alpha_{0}+\alpha_{1}$ educ $_{i}+\alpha_{2}$ cor $_{i}+\alpha_{3}$ urban $_{i}+\alpha_{4}$ morarsozinho $_{i}+\alpha_{5} N_{i}+$ $\alpha_{6} \mathrm{Ne}_{i}+\alpha_{7} \mathrm{Co}_{i}+\alpha_{8}$ Sul $_{i}+\alpha_{9}$ exper $_{i}+\alpha_{9}$ exper $_{i}{ }_{i}+\alpha_{10}$ formal $_{i}+\alpha_{11}$ chefe $_{i}+$ $\sum_{i=12}^{19} \alpha_{i}$ atividade $_{i}+\sum_{i=1}^{n} \quad a_{20+i} Z_{i}+\varepsilon_{i}$

As variáveis são similares às da equação (9), com pequenas modificações. Depr se torna a variável dependente, sendo atribuído valor igual a 1 se o indivíduo foi diagnosticado com depressão e 0 caso contrário; é adicionada a variável morarsozinho, dummy que denota se o indivíduo mora sozinho ou não; são incluídas as variáveis instrumentais $Z$, que determinam se o indivíduo foi diagnosticado ou não com as seguintes doenças: doença de coluna ou costas, artrite ou reumatismo, câncer, diabetes, bronquite ou asma, hipertensão, doença do coração, insuficiência real crônica, tuberculose, tendinite ou tenossinovite, e cirrose; e as dummies de interação entre os setores produtivos e a dummy que denota se o indivíduo foi diagnosticado com depressão não são consideradas.

Assim, é possível resumir as três etapas da estimação da seguinte forma:

1. Estima-se um Probit em D com todas as variáveis exógenas;

2. Obtém-se as probabilidades estimadas;

3. Estima-se a equação de rendimentos do trabalho por MQ2E, utilizando-se apenas o valor estimado da probabilidade como instrumento excluído.

As variáveis utilizadas no último estágio do MQ2E são semelhantes àquelas apresentadas na equação (9), com exceção das dummies de interação supracitadas. O modelo utiliza nesse último estágio o valor estimado das dummies de interação ao invés das mesmas.

\subsection{Fonte e tratamento dos dados}

O presente estudo utiliza a PNAD de 2008 como sua fonte de dados. Tal pesquisa é realizada anualmente pelo Instituto Brasileiro de Geografia e Estatística (IBGE) e aborda diversos aspectos da vida do brasileiro, como renda, escolaridade, trabalho, migração, nupcialidade, saúde, dentre outras características. O IBGE aplica conjuntamente com a PNAD um suplemento em alguns anos. Tal suplemento se relaciona a diversos temas, tais como saúde, crime, acesso à internet e telefone, dentre outros. Utiliza-se a PNAD de 2008 nesse trabalho, pois o suplemento é relacionado à saúde, possuindo questões referentes a diagnósticos e tratamento de diversas doenças.

A PNAD é uma pesquisa baseada em uma amostra complexa, ou seja, estratificada e conglomerada. Assim, o IBGE seleciona aleatoriamente residências a partir de seu registro do Censo Demográfico e atribui a cada uma delas variáveis próprias da amostragem, tais como peso e estrato. Deve-se levar em consideração nas pesquisas todas essas variáveis relacionadas a estratificação, caso contrário, as inferências não são válidas para a população nem para a amostra com suposta reposição simples.

Cabe por fim salientar que as variáveis relacionadas as atividades dos indivíduos (setores produtivos) foram construídas tendo em vista o Código Brasileiro de Ocupações (CBO). Originalmente, o CBO agrega as atividades em doze categorias, quais sejam: agrícolas; indústria de transformação; outras indústrias; construção; comércio e reparação; alojamento e alimentação; transporte, armazenagem e comunicação; administração pública; educação, saúde e serviços sociais; outros serviços coletivos, sociais e pessoais; serviços domésticos e, por 
último, outras atividades 5 . Dado que muitas dessas atividades compartilham características semelhantes, elas foram agrupadas para facilitar a interpretação dos resultados adiante. Assim, as atividades da indústria da transformação, outras indústrias e construção são todas agrupadas sob a denominação Indústria (conforme o próprio CBO o faz). As atividades de alojamento e alimentação e transporte, armazenagem e comunicação foram também agregadas sob uma mesma variável (Transporte). Por fim, as atividades relacionadas a serviços prestados diretamente a pessoas, neste caso as atividades de educação, saúde e serviços sociais e outros serviços coletivos, sociais e pessoais foram também agregados sob a variável Serviços Coletivos.

\section{RESULTADOS}

Inicialmente, foi realizado o teste de endogeneidade de Hausman com o intuito de verificar qual dos métodos de estimação mencionados na seção metodológica é mais adequado. O teste sinalizou a quebra da hipótese de exogeneidade, ou seja, existe relação endógena entre o transtorno depressivo e os rendimentos do trabalho de homens e mulheres. Logo, os coeficientes estimados via MQO são viesados, sendo adequada a estimação através do modelo de MQ2E. Esse resultado para as mulheres está em consonância com o encontrado por Benedicto (2017), no qual a autora também verifica endogeneidade entre o transtorno depressivo e os rendimentos do trabalho. O resultado dos homens, contudo, é discrepante entre este e aquele trabalho. As causas para tal discrepância podem estar relacionadas a diferenças de especificação do modelo, indicando pouca robustez do resultado do teste de Hausman, no caso dos homens. Verifica-se, no entanto, através da Tabela 1, que o os coeficientes estimados para os dois modelos (MQO e MQ2E) e seus respectivos desvios-padrão entre os homens são bastante semelhantes, não interferindo, portanto, nas principais conclusões do trabalho.

Tabela 1: Resultados das estimações dos modelos MQO e de MQ2E.

\begin{tabular}{ccccc}
\hline Variável & $\begin{array}{c}\text { Homens } \\
\text { MQO }\end{array}$ & $\begin{array}{c}\text { Homens } \\
\text { MQ2E }\end{array}$ & $\begin{array}{c}\text { Mulheres } \\
\text { MQO }\end{array}$ & $\begin{array}{c}\text { Mulheres } \\
\text { MQ2E }\end{array}$ \\
\hline Constante & $1,29642^{*}$ & $1,274044^{*}$ & $1,268994^{*}$ & $1,261364^{*}$ \\
& $(0,0175756)$ & $(0,0181284)$ & $(0,0222832)$ & $(0,0234307)$ \\
\hline Educ & $0,104298^{*}$ & $0,1043092^{*}$ & $0,10263^{*}$ & $0,1027295^{*}$ \\
& $(0,0008667)$ & $(0,0008668)$ & $(0,0011156)$ & $(0,0011144)$ \\
\hline Exper & $0,0399315^{*}$ & $0,0401948^{*}$ & $0,0290074^{*}$ & $0,0305252^{*}$ \\
& $(0,0006869)$ & $(0,0006939)$ & $(0,000774)$ & $(0,0007969)$ \\
\hline Exper & $-0,0004679^{*}$ & $-0,0004691^{*}$ & $-0,0002617^{*}$ & $-0,0002766^{*}$ \\
& $(0,0000133)$ & $(0,0000133)$ & $(0,0000168)$ & $(0,0000169)$ \\
\hline Formal & $0,0629418^{*}$ & $0,0617271^{*}$ & $0,1008999^{*}$ & $0,0938552^{*}$ \\
& $(0,0051987)$ & $(0,0052199)$ & $(0,0062485)$ & $(0,0063134)$ \\
\hline Chefe & $0,1317281^{*}$ & $0,1313677^{*}$ & $0,0163096^{* *}$ & $0,0254684^{*}$ \\
& $(0,0056045)$ & $(0,005608)$ & $(0,0066469)$ & $(0,0067374)$ \\
\hline Urban & $0,0996138^{*}$ & $0,1013163^{*}$ & $0,1233251^{*}$ & $01258566^{*}$ \\
& $(0,0085763)$ & $(0,0085932)$ & $(0,0120945)$ & $(0,0120836)$ \\
\hline Branco & $0,1720395^{*}$ & $0,1731253^{*}$ & $0,1501925^{*}$ & $0,1520611^{*}$ \\
& $(0,0052858)$ & $(0,0052941)$ & $(0,0062742)$ & $(0,0062758)$ \\
\hline Sul & $-0,0070263^{*}$ & $-0,00446353$ & 0,0012387 & 0,0136758 \\
& $(0,0072663)$ & $(0,0072839)$ & $(0,0082399)$ & $(0,0083354)$ \\
\hline
\end{tabular}

\footnotetext{
5 O setor de outras atividades é, segundo IBGE (2017), composto por serviços de intermediação financeira, inclusive seguros e previdência privada, atividades imobiliárias, aluguel de veículos, máquinas e objetos pessoais e domésticos, atividades de informática, P\&D das Ciências Humanas e Sociais, serviços prestados a empresas (publicidade, contabilidade, dentre outros) e atividades relacionadas a organismos internacionais e outras instituições extraterritoriais.
} 


\begin{tabular}{|c|c|c|c|c|}
\hline Co & $\begin{array}{l}0,0718152 * \\
(0,0079658)\end{array}$ & $\begin{array}{l}0,0710702 * \\
(0,0079674)\end{array}$ & $\begin{array}{r}0,0224485 * * \\
(0,0094036) \\
\end{array}$ & $\begin{array}{c}0,0248545 \\
(0,0093917)\end{array}$ \\
\hline No & $\begin{array}{c}-0,0747997 * \\
(0,00836)\end{array}$ & $\begin{array}{l}-0,0790564 * \\
(0,0084496)\end{array}$ & $\begin{array}{c}-0,0895064^{*} \\
(0,010425)\end{array}$ & $\begin{array}{l}-0,1004442 * \\
(0,0105688)\end{array}$ \\
\hline $\mathrm{Ne}$ & $\begin{array}{l}-0,3373212 * \\
(0,0065588)\end{array}$ & $\begin{array}{l}-0,340325^{*} \\
(0,0066244)\end{array}$ & $\begin{array}{l}-0,3304253^{*} \\
(0,0078706)\end{array}$ & $\begin{array}{l}-0,3382629 * \\
(0,0079826)\end{array}$ \\
\hline Agricultura & $\begin{array}{l}-0,338648^{*} \\
(0,0128168)\end{array}$ & $\begin{array}{l}-0,316901^{*} \\
(0,014455)\end{array}$ & $\begin{array}{l}-0,3674318^{*} \\
(0,0254864)\end{array}$ & $\begin{array}{l}-0,4109491 * \\
(0,0338655)\end{array}$ \\
\hline Indústria & $\begin{array}{l}-0,1012665^{*} \\
(0,0096846)\end{array}$ & $\begin{array}{l}-0,0753955^{*} \\
(0,0111324)\end{array}$ & $\begin{array}{l}-0,3213977 * \\
(0,0133108)\end{array}$ & $\begin{array}{l}-0,2869259 * \\
(0,0167694)\end{array}$ \\
\hline Comércio & $\begin{array}{l}-0,1769269 * \\
(0,0103284)\end{array}$ & $\begin{array}{l}-0,151839 * \\
(0,0120108)\end{array}$ & $\begin{array}{l}-0,2613936^{*} \\
(0,0126508)\end{array}$ & $\begin{array}{l}-0,2652547 * \\
(0,0156235)\end{array}$ \\
\hline Serviços Coletivos & $\begin{array}{l}0,029738 * * \\
(0,013615)\end{array}$ & $\begin{array}{l}0,0604348 * \\
(0,0160685)\end{array}$ & $\begin{array}{r}-0,0492076 * \\
(0,0119394)\end{array}$ & $\begin{array}{l}-0,0208001 \\
(0,0149202)\end{array}$ \\
\hline Serviço Doméstico & $\begin{array}{c}-0,3727292 * \\
(0,023469)\end{array}$ & $\begin{array}{c}-0,3186007 * \\
(0,027462)\end{array}$ & $\begin{array}{r}-0,3943968^{*} \\
(0,0131794)\end{array}$ & $\begin{array}{l}-0,4302087^{*} \\
(0,0163845)\end{array}$ \\
\hline Transportes & $\begin{array}{l}-0,1265691 * \\
(0,0112424)\end{array}$ & $\begin{array}{l}-0,1047983^{*} \\
(0,0129877)\end{array}$ & $\begin{array}{c}-0,2522375^{*} \\
(0,015219)\end{array}$ & $\begin{array}{c}-0,2390815^{*} \\
(0,0191426)\end{array}$ \\
\hline Administração Pública & $\begin{array}{l}0,1406046^{*} \\
(0,0132513)\end{array}$ & $\begin{array}{l}0,1645339 * \\
(0,0157715)\end{array}$ & $\begin{array}{c}0,1471918^{*} \\
(0,016951)\end{array}$ & $\begin{array}{c}0,145^{*} \\
(0,0213238)\end{array}$ \\
\hline Agricultura*Depressão & $\begin{array}{c}-0,1048695 * * * \\
(0,0546222)\end{array}$ & $\begin{array}{l}-0,3164747 \\
(0,2220522)\end{array}$ & $\begin{array}{l}-0,0425182 \\
(0,0910281)\end{array}$ & $\begin{array}{c}0,2184256 \\
(0,3083804)\end{array}$ \\
\hline Indústria*Depressão & $\begin{array}{l}-0,0822777 * \\
(0,0284585)\end{array}$ & $\begin{array}{l}-0,5486163 * \\
(0,1449875)\end{array}$ & $\begin{array}{l}-0,127138 * \\
(0,039073)\end{array}$ & $\begin{array}{l}-0,9101503^{*} \\
(0,1200999)\end{array}$ \\
\hline Comércio*Depressão & $\begin{array}{l}-0,0841983 \\
(0,0590583)\end{array}$ & $\begin{array}{c}-0,4745254 * * \\
(0,221846) \\
\end{array}$ & $\begin{array}{l}-0,0517426 \\
(0,0379905)\end{array}$ & $\begin{array}{c}-0,2372332 * * * \\
(0,1240999) \\
\end{array}$ \\
\hline Transportes*Depressão & $\begin{array}{l}-0,124881 * * \\
(0,0557804)\end{array}$ & $\begin{array}{l}-0,3705078 \\
(0,2372368)\end{array}$ & $\begin{array}{r}-0,2379835^{*} \\
(0,0517303)\end{array}$ & $\begin{array}{c}-0,7657792^{*} \\
(0,18317)\end{array}$ \\
\hline $\begin{array}{c}\text { Serviços } \\
\text { Coletivos*Depressão }\end{array}$ & $\begin{array}{l}-0,0635545 \\
(0,0688221)\end{array}$ & $\begin{array}{c}-0,5425112 * * * \\
(0,2887331)\end{array}$ & $\begin{array}{l}-0,1155312^{*} \\
(0,0224538)\end{array}$ & $\begin{array}{l}-0,8141905^{*} \\
(0,0887577)\end{array}$ \\
\hline $\begin{array}{c}\text { Serviço } \\
\text { Doméstico*Depressão }\end{array}$ & $\begin{array}{l}-0,2492806 \\
(0,1892297)\end{array}$ & $\begin{array}{c}-1,762237 * * \\
(0,6894375)\end{array}$ & $\begin{array}{c}-0,026387 \\
(0,0224538)\end{array}$ & $\begin{array}{c}0,1538451 \\
(0,0963336)\end{array}$ \\
\hline $\begin{array}{c}\text { Administração } \\
\text { Pública*Depressão }\end{array}$ & $\begin{array}{r}-0,0420193 \\
(0,068651)\end{array}$ & $\begin{array}{l}-0,2851221 \\
(0,3056905)\end{array}$ & $\begin{array}{l}-0,12351 * * \\
(0,0547161)\end{array}$ & $\begin{array}{c}-0,3222563 * * \\
(0,1777265)\end{array}$ \\
\hline $\begin{array}{c}\text { Outras } \\
\text { atividades*depressão }\end{array}$ & $\begin{array}{l}-0,0577194 \\
(0,0676143)\end{array}$ & $\begin{array}{c}0,7982883 * * \\
(0,3140434)\end{array}$ & $\begin{array}{r}-0,1309855^{*} \\
(0,0497483)\end{array}$ & $\begin{array}{c}-0,3797488 * * \\
(0,1789246)\end{array}$ \\
\hline Número de observações & 92881 & 92881 & 65483 & 65483 \\
\hline $\mathbf{R}^{2}$ & 0,4192 & 0,4193 & 0,3960 & 0,3974 \\
\hline VIF & 2,45 & 2,81 & 2,35 & 2,99 \\
\hline
\end{tabular}

Fonte: Elaboração própria.

Nota: * denota significância a 1\%; ** denota significância a 5\%; *** denota significância a $10 \%$.

Como apenas os coeficientes do modelo MQ2E são consistentes no caso tanto dos homens quanto das mulheres, analisa-se os resultados encontrados apenas a partir desse método de estimação. Inicialmente, são salientados os resultados das variáveis de interação entre os setores de atividades dos indivíduos e o diagnóstico do transtorno depressivo, que é o foco do presente estudo. $\mathrm{O}$ coeficiente da variável de interação entre o diagnóstico do transtorno depressivo e agricultura não é significativa estatisticamente, indicando, portanto, que um homem, diagnosticado com essa doença, alocado neste setor, tem rendimento semelhante a um agricultor sem o transtorno depressivo. Outros coeficientes de interação não significativos são os das interações do transtorno depressivo com os setores de transportes e comunicação e administração pública.

Logo, a incidência do transtorno depressivo dos homens empregados nos setores da indústria, comércio, serviços coletivos, serviços domésticos e outras atividades é significativa e faz com que seus rendimentos variem em $-54,86 \%,-47,45 \%,-54,25 \%,-176,22 \%$ e $79,82 \%$ em comparação aos rendimentos de homens empregados nestes mesmos setores, mas sem 
diagnóstico de transtorno depressivo. É importante ressaltar a diferenciação da queda dos rendimentos entre os setores produtivos, o que justifica a separação das atividades realizada. Caso o percentual de queda dos rendimentos dos indivíduos dos oito setores utilizados no trabalho fosse estatisticamente idêntico, então essa separação seria desnecessária. Percebeu-se, contudo, que há quedas de rendimentos da ordem de 176,22\% (serviço doméstico), até situações em que os rendimentos dos indivíduos aumentam em 79,82\% (outras atividades). Obviamente, o transtorno depressivo afeta indivíduos de formas distintas e os resultados corroboram que essa doença tem impactos setoriais também diferenciados.

Em relação às mulheres, tem-se que o coeficiente da variável de interação entre o diagnóstico do transtorno depressivo e agricultura também não é significativo, indicando, portanto, que uma mulher, diagnosticada com a doença e alocada no setor agrícola tem rendimento semelhante àquela com depressão alocada no mesmo setor. Outro coeficiente de interação não significativo é o da interação entre o acometimento pelo transtorno depressivo e o setor de serviço doméstico.

Assim, os coeficientes de interação do transtorno depressivo com os setores da indústria, comércio, transporte e comunicação, serviços coletivos, administração pública e outras atividades são significativos implicam em variações de $-91,02 \%,-23,72 \%,-76,58 \%,-81,42 \%$, $-32,23 \%$ e $-37,97 \%$ em comparação aos rendimentos de mulheres empregadas nestes mesmos setores, mas sem o diagnóstico do transtorno depressivo. Novamente, percebe-se que o impacto setorial do transtorno depressivo sobre as mulheres é assim como no caso dos homens, diferenciado. Nesse sentido, o diagnóstico da doença reduz os rendimentos das mulheres empregadas na indústria em aproximadamente $91 \%$ e em 32,23\% daquelas que trabalham na administração pública. Naturalmente, essa diferenciação em termos de queda/elevação dos rendimentos e em diversas magnitudes motiva a separação das mulheres entre os oito setores utilizados no presente estudo.

Dado o ineditismo do presente estudo, desconhece-se trabalhos que tenham verificado o impacto do transtorno depressivo e do setor de atividade do indivíduo sobre seus rendimentos. Isso torna difícil a comparação dos resultados esboçados acima a qualquer outro percentual, dado o desconhecimento desses possíveis outros coeficientes

Com relação às demais variáveis de controle, verifica-se que os coeficientes de educação, experiência e experiência ao quadrado foram todos significativos a $1 \%$, em conformidade com a teoria do capital humano (RESENDE e WILLYE (2006). Assim, é possível concluir que o aumento de 1 ano de escolaridade eleva os rendimentos dos homens e mulheres em respectivamente 10,43\% e 10,27\%. Ao mesmo tempo, o aumento de 1 ano de experiência eleva os rendimentos dos homens e mulheres em 4,02\% e 3,05, respectivamente.

As variáveis formal, chefe, urban e branco foram também significativas a $1 \%$ tanto para homens quanto para mulheres. Assim, por exemplo, o fato de o indivíduo trabalhar no mercado de trabalho formal implica em aumento nos rendimentos da ordem de 6,17\% e 9,39\%, respectivamente. A formalização das relações de trabalho facilita a negociação de rendimentos entre empregado e patrão, favorecendo, assim, maiores remunerações para os empregados formalizados. $\mathrm{O}$ indivíduo chefe de família também possui bônus em seu salário da ordem de $13,14 \%$ e $2,55 \%$ no caso deste ser homem ou mulher, respectivamente. Dado que o chefe de família tende a ser o principal provedor da família, tal resultado condiz com essa expectativa (HAUSSMANN e GOLGHER, 2016). Continuando a análise, homem ou mulher residentes na zona urbana têm bônus salarial da ordem de $10,13 \%$ e $12,58 \%$, respectivamente. Tal resultado é justificado pelo maior dinamismo econômico da zona urbana, favorecendo, portanto, maiores remunerações para os homens (RESENDE e WILLYE (2006).

As variáveis regionais foram todas significativas a $1 \%$, tendo como referência a região Sudeste, excetuando-se aquela referente à região Sul para os homens e Sul e Centro-Oeste no 
caso das mulheres. Assim, caso um homem resida na região Centro-Oeste, seus rendimentos do trabalho se elevam em 7,11\%. Por outro lado, homens e mulheres residentes nas regiões Norte e Nordeste têm seus rendimentos diminuídos em 7,91\%, 34,93\%, 10,04\% e 33,83\%, respectivamente. Benedicto (2017) verificou que todas as variáveis regionais foram significativas a $1 \%$, com as variáveis referentes às regiões Sul, Nordeste, Norte e Centro-Oeste implicando em diminuições de 29,44\%, 62,09\%, 27,94\% e 13,93\%, respectivamente, nos rendimentos das mulheres residentes nessas regiões.

Analisando-se a seguir as variáveis relacionadas aos setores de atividades dos homens, tem-se que que todos os coeficientes são significativos, ou seja, há diferenças entre os rendimentos dos indivíduos nos setores da agricultura, indústria, comércio, serviços coletivos, serviços domésticos, transportes e comunicação e administração púbica em relação ao setor de outras atividades (referência). Assim, caso um homem trabalhe no setor da agricultura, seu salário é 31,69\% menor que aquele que trabalha no setor referente a outras atividades. Por outro lado, caso ele trabalhe nos setores da indústria, comércio, serviços domésticos e transporte e comunicação, seu salário é $7,54 \%, 15,18 \%, 31,86 \%$ e 10,48\% menor que o trabalhador do referido setor. Por fim, os rendimentos dos homens que trabalham nos setores de serviços coletivos e administração pública são 6,04\% e 16,45\% mais elevados que suas contrapartes no setor de outras atividades, respectivamente.

Em relação às mulheres, todas as variáveis setoriais foram significativas. Caso uma mulher trabalhe no setor da agricultura, seu salário é, em média, 41,09\% menor em relação à sua contraparte no setor de outras atividades. Por outro lado, caso ela trabalhe nos setores da indústria, comércio, serviços domésticos e transporte e comunicação, seu rendimento é 28,69\%, $26,53 \%, 43,02 \%$ e $23,91 \%$ menor aquela do setor de outras atividades. Por fim, caso a mulher trabalhe no setor da administração pública, seu salário é $14,5 \%$ maior que uma trabalhadora produtivamente semelhante no setor de outras atividades. O coeficiente da variável do setor de serviços coletivos não foi significativo, indicando, portanto, que os rendimentos de duas mulheres semelhantemente produtivas, uma nesse setor e a outra em outras atividades, são na média idênticos.

\section{CONSIDERAÇÕES FINAIS}

O presente estudo teve como objetivo analisar o impacto do transtorno depressivo sobre os rendimentos de homens e mulheres, tendo em vista seus setores produtivos. Conclui-se que o fato de o indivíduo ter sido diagnosticado com transtorno depressivo diminui seus rendimentos em todos os setores analisados, exclusive o de outras atividades.

Além disso, foi possível concluir que o transtorno depressivo é catalisado por diversas variáveis, tais como região de residência (Sul), cor, papel desempenhado no domicílio, o fato de o indivíduo morar sozinho, idade e, principalmente, possíveis doenças crônicas que o acometam. Além disso, a regularidade empírica de duas mulheres para cada homem com essa enfermidade é mantida ou ampliada em diversas situações, enfatizando a suscetibilidade maior das primeiras em relação à doença.

É importante salientar a contribuição do estudo na literatura ao concluir que a relação existente entre os rendimentos dos indivíduos e o transtorno depressivo não é exógena, fazendo com que a relação causal parcial seja de difícil apuração apenas pelo uso do modelo MQO, o que demandou a utilização do método de MQ2E.

Outro diferencial do estudo é permitir que o impacto do transtorno depressivo sobre seus rendimentos variasse de acordo com o setor no qual homens e mulheres se encontram empregados. Assim, os resultados denotam a causalidade parcial da interação entre o transtorno depressivo, o setor de atividade e os rendimentos dos indivíduos. Como exemplo, verificou-se 
que a mulher empregada no setor da administração pública e diagnosticada com o transtorno depressivo tem um salário 32,23\% menor que sua contraparte não diagnosticada com essa doença.

Os resultados fornecem subsídios para a elaboração de políticas públicas, com o objetivo de diminuir a incidência do transtorno depressivo sobre homens e mulheres de diferentes setores. Além disso, ficou evidente que a doença implica na diminuição de rendimentos na maioria dos setores, o que implica em prejuízos para os indivíduos, a nível micro, e para o país, do ponto de vista macroeconômico. Assim, por exemplo, funcionários públicos precisam de políticas de prevenção mais bem desenhadas e focadas, dada a maior incidência do transtorno depressivo em ambos os gêneros nesse setor em comparação àqueles oriundos de outros setores.

Por fim, ressalta-se que o presente trabalho também possui algumas limitações, que incluem a indisponibilidade de dados em relação aos indivíduos com o transtorno depressivo, mas sem o diagnóstico da doença. Isso faz com que os dados utilizados possam subestimar a parcela da população brasileira acometida pela enfermidade.

\section{REFERÊNCIAS}

AUBE, J.; FLEURY, J.; SMETANA, J. - Changes in women's roles: impact on and social policy implications for the mental health of women. Dev Psychopathol.12:633-56, 2000.

BAPTISTA, M. N., BAPTISTA, A. S. D.; OLIVEIRA, M. D. G. D. (1999). Depressão e gênero: por que as mulheres deprimem mais que os homens?. Temas em Psicologia, 7(2), 143-156. 1999.

BENEDICTO, B. V. O efeito do transtorno depressivo sobre os rendimentos do trabalho no Brasil, 2017. Dissertação de mestrado.

BÓS, M. G. A.; BÓS, J. G. Â. Fatores determinantes e conseqüências econômicas da depressão entre os idosos no Brasil, Revista Brasileira de Ciências do Envelhecimento Humano, v.2, n.2 2005.

CAI, L.; KALB, G. Health status and labour force participation evidence from Australia. Health Economics. 2006, 15 (3) : 241-61.

CIRINO, J. F.; LIMA, J. E. Diferenças de rendimentos entre as regiões metropolitanas de Belo Horizonte e Salvador: uma discussão a partir da decomposição Oaxaca-Blinder. Revista Econômica do Nordeste. Vol. 43, n. 2, Abril-Junho, 2012.

CORREIA, K. M. L.; BORLOTI, E. Mulher e depressão: Uma análise comportamental contextual. acta comportamentalia, 19(3), 359-373, 2011.

GROSSMAN, M. On the concept of health capital and the demand of health. The Journal of Political Economy, vol. 80, n. 2, Mar.-Apr., p. 223-255, 1972.

HAUSMAN, J.A. Specification Tests in Econometrics. Econometrica. Vol. 46, n. 6, Nov. 1978, pp. 1251-1271, 1978. 
HAUSSMANN, S.; GOLGHER, A. B. Shrinking gender wage gaps in the Brazilian labor market: an application of the APC approach. Nova Economia, Belo Horizonte, vol. 26, n. 2, p. 429-464, 2016.

INSTITUTO BRASILEIRO DE GEOGRAFIA E ESTATÍSTICA. Pesquisa Nacional de Amostragem de Domícilios: PNAD 2008.

JUSTO, L. P.; CALIL, H. M. Depressão: o mesmo acometimento para homens e mulheres. Revista Psiquiatria Clínica, 33(2), 74-9, 2006.

LEDERBOGEN, F., KIRSCH, P., HADDAD, L., STREIT, F., TOST, H., SCHUCH, P.; MEYER- LINDENBERG, A. City living and urban upbringing affect neural social stress processing in humans. Nature, 474(7352), 498-501,2011.

LUCAS, R. E. On the mechanics of economic development. Journal of Monetary Economics, vol. 22, 3-42, 1988.

MANKIW, N. G.; ROMER, D.; WEIL, D. A contribution to the empirics of economic growth. Quarterly Journal of Economics, vol. 107(2), 407-437, 1992.

MINCER, J. Scholling, experience, and eranings. National Bureau of Economic Reasearch. 152 p. 1974.

RESENDE. M.; WYLLIE, R. Retornos para educação no Brasil: evidências empíricas adicionais. Economia Aplicada, vol. 10, 349-365, 2006.

ROMER, P. Endogenous technological change. Journal of Political Economy, 99(5, pt. II), S71-S102, 1990.

SANTOS, M. J.; KASSOUF, A. L. Uma investigação dos determinantes socioeconômicos da depressão mental no Brasil com ênfase nos efeitos da educação. Econ. Aplic. São Paulo, v. 11, n. 1, p. 5-26, 2007.

SOBOCKI, P.; JÖNSSON, B. ; ANGST, J. ; REHNBERG, C. Cost of depression in Europe. The Journal of Mental Health Policy and Economics, 2006.

WOLFF, E. N. Poverty and Income Distribution, 2nd ed. West Sussex: Wiley Blackwell, 2009.

WOOLDRIDGE, M.J. Econometric Analysis of cross section and panel data. MIT Press, 2002.

WORLD HEALTH ORGANIZATION (WHO). Depression and Other Common Mental Disorders: Global Health Estimates. Geneva, 2017.

Recebido: 28/07/2020

Aceito: 23/04/2021

Publicado: Agosto de 2021 